\title{
New concepts in the management of retinal vein occlusions
}

Abbreviations: PGIMER, post graduate institute of medical education and research; CRVO, central retinal vein occlusion; BRVO, branched retinal vein occlusion; VEGF, vascular endothelial growth factor; US-FDA, us food and drug administration

\section{Opinion}

The two major types of retinal vein occlusion are branched retinal vein occlusion (BRVO) and central retinal vein occlusion (CRVO). Five-year incidence of BRVO is $0.6 \%$ and CRVO is $0.2 \% .{ }^{1}$ Macular edema secondary to retinal vein occlusions is an important cause of vision loss. The management of RVO has been revolutionised ever since the introduction of intravitreal pharmacotherapy including the use of anti-vascular endothelial growth factor (VEGF) agents. Recently, anti-VEGFs and corticosteroids have been found to be safe and efficacious in the management of retinal vein occlusions.

\section{Anti-VEGF therapy: newer advances}

Retinal vein occlusion leads to increased expression of VEGF in the ocular milieu which is responsible for retinal neovascularisation and increase in retinal vascular permeability. This results in breakdown of the blood-retinal-barrier and subsequent development of macular edema. All three currently available anti-VEGF agents (ranibizumab, bevacizumab and aflibercept) have been successfully used in management of macular edema secondary to retinal vein occlusions. The CRUISE and BRVO studies compared two different doses of ranibizumab $(0.3 \mathrm{mg}$ versus $0.5 \mathrm{mg})$ to sham injections in patients with macular edema secondary to retinal vein occlusions and showed significant improvement in visual acuity in treated group versus the control group. ${ }^{2,3}$ This led to the US Food and Drug Administration (US-FDA) approval of ranibizumab $(0.3 \mathrm{mg})$ for the treatment of macular edema secondary to retinal vein occlusions in 2010. In the VIBRANT trial, monthly intravitreal aflibercept injections provided significantly greater visual benefit compared with macular grid laser in eyes with macular edema secondary to BRVO. ${ }^{4}$ Aflibercept was approved for the management of macular edema following CRVO in September 2012. Thus, introduction of anti-VEGF agents has been considered as the beginning of a new era in the treatment of macular edema secondary to retinal vein occlusions as these agents target the disease at the molecular level.

\section{Corticosteroids: current role}

Corticosteroids have a role in the treatment of macular edema secondary to retinal vein occlusions because of their anti-inflammatory and anti-angiogenic properties. There has been an interest in the use of local corticosteroid therapy in the management of both BRVO and CRVO. The SCORE study compared two different doses of intravitreal triamcinolone (1mg and $4 \mathrm{mg}$ ) to the standard of scare which is observation in CRVO and macular laser treatment in BRVO. SCORECRVO study concluded that patients in corticosteroid group are five times more likely to have substantial visual gain in visual acuity as compared to observation group. The SCORE-BRVO study concluded that corticosteroid and macular laser treatment has similar impact on vision. ${ }^{5,6}$ The GENEVA study evaluated the use of sustained-release
Volume 6 Issue $6-2017$

\author{
Sabia Handa, Aniruddha Agarwal, Jagat Ram \\ Department of Ophthalmology, Post Graduate Institute of \\ Medical Education and Research (PGIMER), India
}

Correspondence: Jagat Ram, Professor, Department of Ophthalmology Advanced Eye Centre Post Graduate Institute of Medical Education and Research (PGIMER), Chandigarh-1600 I2, India, Email drjagatram@gmail.com

Received: April 28, 2017 | Published: April 28, 2017

dexamethasone implant in the management of macular edema due to retinal vein occlusions. ${ }^{7}$ Based on the results of the GENEVA trial, intravitreal dexamethasone implant (OZURDEX $\left.{ }^{\circledR}\right)$ received FDA approval for the treatment of macular edema secondary to retinal vein occlusions in 2009.

\section{Future directions}

Recently, a number of trials have been initiated with an attempt to reduce the treatment burden by employing a combination of the available therapies. In the recently published WAVE trial, ultra-wide field fluorescein angiography was performed among patients with retinal vein occlusions. The study evaluated the use of wide field image-guided peripheral retinal photocoagulation (of the targeted nonperfused retina) combined with ranibizumab. However, the results of the study did not show any significant reduction in the total number of intravitreal injections required over a period of 12 months. ${ }^{8}$ Similarly, the RELATE trial evaluated higher dose of ranibizumab $(2.0 \mathrm{mg})$ and laser therapy in the management of macular edema in retinal vein occlusions. The results showed that higher dose of ranibizumab and laser therapy offered no additional benefits in eyes with retinal vein occlusions. ' A number of newer agents are being studied for the management of retinal vein occlusions. A sustained release implant, IBI-20089 is a novel drug product based on Verisome drug delivery platform technology with different doses of triamcinolone. It is being evaluated for its role in patients with chronic cystoid edema due to retinal vein occlusions. ${ }^{10}$ As there is progressive visual impairment due to degeneration of photoreceptors in eyes with retinal vein occlusions, stem cell therapy is currently being investigated for the treatment of macular edema in BRVO and CRVO. ${ }^{11}$

\section{Acknowledgements}

None.

\section{Conflicts of interest}

The authors declare there are no conflicts of interest.

\section{References}

1. Klein R, Klein BE, Moss SE, et al. The epidemiology of retinal vein occlusion: the Beaver Dam Eye Study. Trans Am Ophthalmol Soc. 2000;98:133-141. 
2. Brown DM, Campochiaro PA, Singh RP, et al Ranibizumab for macular edema following central retinal vein occlusion: six -month primary end point results of a phase III study. Ophthalmology. 2010;117(6):1124-1133.

3. Campochiaro PA, Heier JS, Feiner L, et al. BRAVO Investigators Ranibizumab for macular edema following branch retinal vein occlusion: six -month primary end point results of a phase III study. Ophthalmology. 2010;117(6):1102-1112.

4. Campochiaro PA, Clark WL, Boyer DS, et al. Intravitreal aflibercept for macular edema following branch retinal vein occlusion: the 24 -week results of the VIBRANT study. Ophthalmology. 2015;122(3):538-544.

5. Scott IU, Ip MS, VanVeldhuisen PC, et al. A randomized trial comparing the efficacy and safety of intravitreal triamcinolone with standard care to treat vision loss associated with macular edema secondary to branch retinal vein occlusion. Arch Ophthalmol. 2009;127(9):1115-1128.

6. Ip MS, Scott IU, VanVeldhuisen PC, et al. A randomized trial comparing the efficacy and safety of intravitreal triamcinolone with observation to treat vision loss associated with macular edema secondary to central retinal vein occlusion. Arch Ophthalmol. 2009;127(9):1101-1114.
7. Haller JA, Bandello F, Belfort R, et al. Randomized, sham-controlled trial of dexamethasone intravitreal implant in patients with macular edema due to retinal vein occlusion. Ophthalmology. 2010;117(6):1134-1146.

8. Wykoff CC, Ou WC, Wang R, et al. Peripheral Laser for Recalcitrant Macular Edema Owing to Retinal Vein Occlusion: The WAVE Trial. Ophthalmology. 2017;124(6):919-921.

9. Campochiaro PA, Hafiz G, Mir TA, et al. Scatter Photocoagulation Does Not Reduce Macular Edema or Treatment Burden in Patients with Retinal Vein Occlusion: The RELATE Trial. Ophthalmology. 2015;122(7):1426-1437.

10. Fung AE. A novel sustained -release intravitreal drug delivery system for retinal vascular disease. Retina Today. 2010;5:51 -53.

11. Clinical trial of autologous intravitreal bone -marrow CD34+ stem cells for retinopathy. 